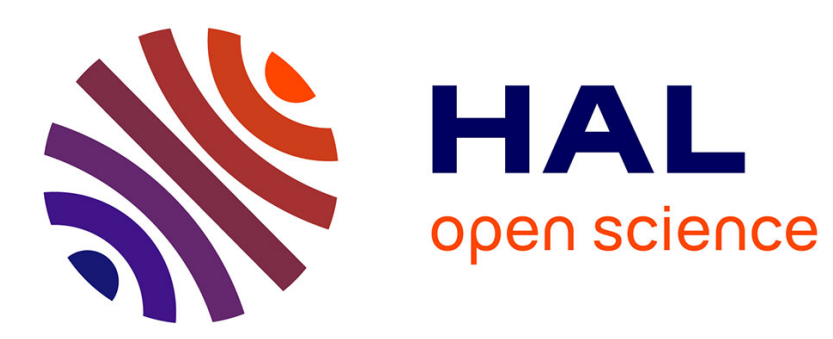

\title{
Propagation d'une onde infrarouge guidée en présence de porteurs libres
}

\author{
A. Azema, J. Botineau, F. Gires, A. Saïssy, C. Vanneste
}

\section{To cite this version:}

A. Azema, J. Botineau, F. Gires, A. Saïssy, C. Vanneste. Propagation d'une onde infrarouge guidée en présence de porteurs libres. Revue de Physique Appliquée, 1976, 11 (2), pp.239-243. 10.1051/rphysap:01976001102023900 . jpa-00244053

\section{HAL Id: jpa-00244053 https://hal.science/jpa-00244053}

Submitted on 1 Jan 1976

HAL is a multi-disciplinary open access archive for the deposit and dissemination of scientific research documents, whether they are published or not. The documents may come from teaching and research institutions in France or abroad, or from public or private research centers.
L'archive ouverte pluridisciplinaire HAL, est destinée au dépôt et à la diffusion de documents scientifiques de niveau recherche, publiés ou non, émanant des établissements d'enseignement et de recherche français ou étrangers, des laboratoires publics ou privés. 
Classification Physics Abstracts

8.860

\title{
PROPAGATION D'UNE ONDE INFRAROUGE GUIDÉE EN PRÉSENCE DE PORTEURS LIBRES
}

\author{
A. AZEMA, J. BOTINEAU, F. GIRES, A. SAÏSSY et C. VANNESTE \\ Laboratoire de Physique de la Matière Condensée (*), \\ Parc Valrose, 06034 Nice, France
}

(Reçu le 4 avril 1975, révisé le 27 juin 1975, accepté le 23 octobre 1975)

\begin{abstract}
Résumé. - Les modes de propagation d'un guide d'onde $10,6 \mu \mathrm{m}$ de surface en arséniure de gallium sont profondément perturbés par l'injection de photoporteurs. Une étude théorique du phénomène est présentée, ainsi que quelques résultats expérimentaux.

Abstract. - The modes of propagation of a gallium arsenide $10.6 \mu \mathrm{m}$ slab wave guide are strongly disturbed by injection of photocarriers. A theoretical study of the phenomenon and some experimental results are presented.
\end{abstract}

1. Introduction. - L'introduction de porteurs libres dans un milieu primitivement isolant apporte à la constante diélectrique complexe de ce milieu une perturbation qui peut être considérable. Au voisinage de la fréquence de plasma, l'indice de réfraction varie d'un ordre de grandeur, variation qui s'accompagne de l'apparition d'une absorption importante (Fig. 1) [1].

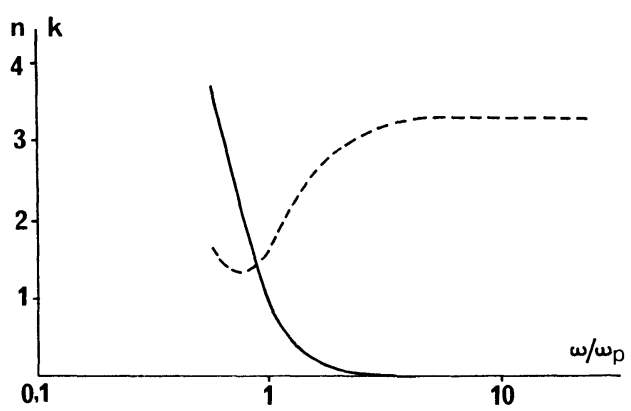

Fig. 1. - Variation théorique de l'indice de réfraction $n$ et du coefficient d'extinction $k$ en fonction de $\omega / \omega_{\mathrm{p}}$ pour GaAs. $\omega_{\mathrm{c}}=5 \times 10^{13} \mathrm{rad}_{\mathrm{s}} \mathrm{s}^{-1}$ (trait plein : $k$, pointillé : $n$ ).

Il peut être tentant, et c'est là tout l'intérêt de la question, d'utiliser ces grandes variations de constantes optiques comme moyens électrooptiques pour moduler en amplitude ou en phase une onde optique. En effet, les moyens électrooptiques usuels ne perturbent, au mieux, que la $3^{\mathrm{e}}$ décimale de l'indice de réfraction, alors que nous pouvons atteindre ici l'indice lui-même.

D'autre part, les porteurs sont sensibles aux champs électriques et magnétiques, à la pression, à la tempé-

(*) Associé au C. N. R. S. n 190. rature, etc... Il doit donc être facile d'en modifier, de l'extérieur, la densité.

Nous nous proposons donc de rechercher la perturbation apportée à la propagation d'une onde optique dans un milieu dont on peut contrôler de l'extérieur la densité ou la répartition de porteurs libres.

Malheureusement, il est très difficile, à l'aide d'une contrainte extérieure, de modifier, rapidement, dans la masse, la densité de charges libres. Il est par contre très facile, par des moyens électriques ou optiques, de modifier à la surface d'un semi-conducteur la concentration des porteurs de charges. Cette modification n'intéressant que la surface du cristal, nous sommes conduits à étudier la propagation d'une onde optique de surface, dans le spectre infrarouge, c'est-à-dire à fréquence proche de la fréquence de plasma, à l'intérieur de la bande interdite d'un semi-conducteur.

Nous étudions ici, sous ses aspects théorique et expérimental, l'interaction d'une onde infrarouge guidée à la surface d'un monocristal d'AsGa avec des porteurs injectés optiquement.

2. Principes de l'étude. - Le guide de lumière infrarouge est obtenu en épitaxiant sur un cristal d'AsGa fortement dopé $\left(n^{+}=2 \times 10^{18} \mathrm{~cm}^{-3}\right)$, une couche d'épaisseur $16 \mu \mathrm{m}$ d'AsGa peu dopé $\left(n=1,5 \times 10^{15} \mathrm{~cm}^{-3}\right)$. Cette variation de dopage conduit à une variation d'indice suffisante (Fig. 1) pour guider une onde de longueur d'onde à $10,6 \mu \mathrm{m}$ provenant d'un laser à $\mathrm{CO}_{2}$. Le couplage entre le faisceau du laser à $10,6 \mu \mathrm{m}$ et le guide d'onde est obtenu par un prisme de Germanium. Un laser à rubis déclenché (impulsions de durée $40 \times 10^{-9} \mathrm{~s}$ ), convenablement atténué, éclaire latéralement la couche d'AsGa et y 
induit des paires électrons trous sur une profondeur inférieure à $0,5 \mu \mathrm{m}$.

3. Modèles théoriques. - L'étude consiste à rechercher les solutions rigoureuses des équations de Maxwell dans la structure infinie, stratifiée, constituée par (Fig. 2) :

- un substrat semi-conducteur 1 fortement dopé $n$,

- une couche épitaxiée 2 du même semi-conducteur, d'épaisseur $a$, de dopage initial faiblement $n$ et dans laquelle on induit de l'extérieur $(z=0)$ une certaine quantité de porteurs caractérisés par une densité volumique en surface $N_{2}^{\prime}$ et une répartition exponentielle de constante d'espace $\gamma$,

— un milieu semi-indéfini 3 (air ou vide).

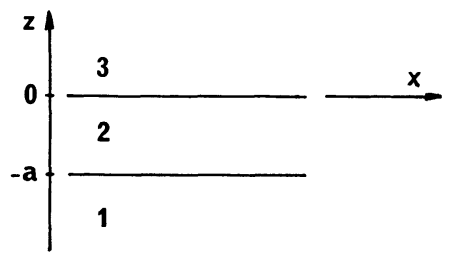

Fig. 2. - Schéma du guide. $x$ Direction de propagation. 1) GaAs $n^{+}$. 2) GaAs n. 3) Air.

Le calcul général est donné en annexe et les résultats appliqués à la structure étudiée expérimentalement sont présentés figure 3.

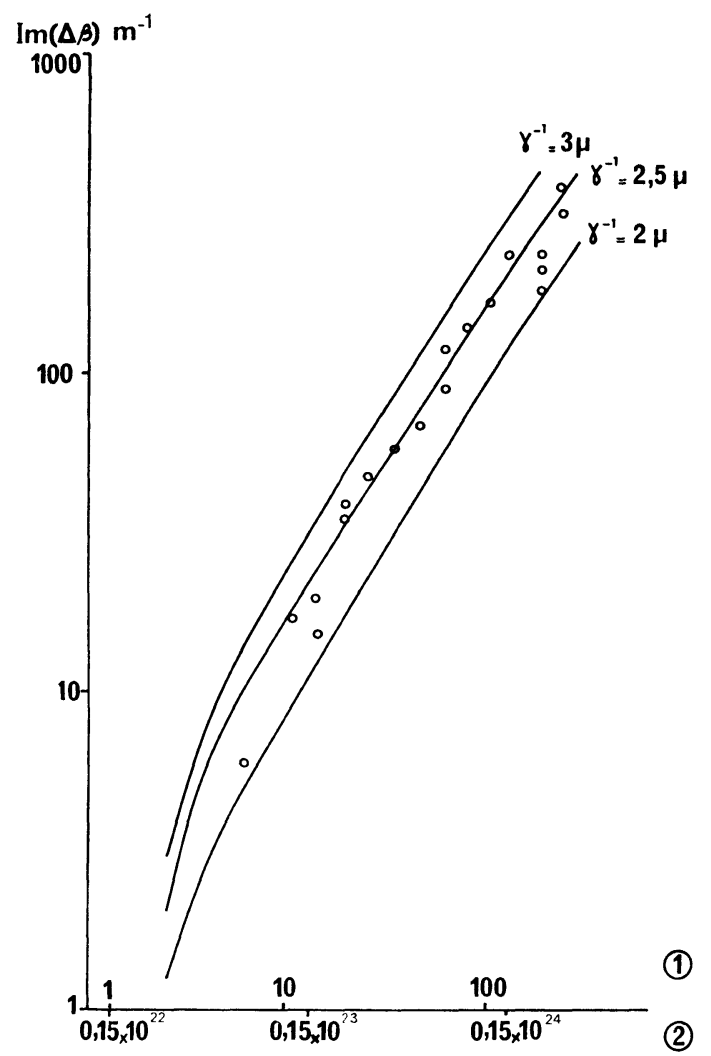

FIg. 3. - Evolution de la partie imaginaire de la constante de propagation $\beta$ avec la concentration des photoporteurs pour 3 valeurs de $\gamma$. 1) Intensité excitatrice (échelle arbitraire).

2) Concentration volumique en surface de la couche.
Avant perturbation, on n'observe pratiquement que les modes fondamentaux $\mathrm{TE}_{0}$ et $\mathrm{TM}_{0}$.

La répartition d'énergie dans ces deux modes est indiquée sur la figure 4. La quasi-totalité de l'énergie est confinée dans la couche épitaxiée. Une très petite queue exponentielle dans l'air permet leur couplage avec une onde extérieure (le mode TE est plus facile à coupler). Une légère pénétration dans le substrat est responsable de l'essentiel des pertes de propagation de la structure non perturbée.

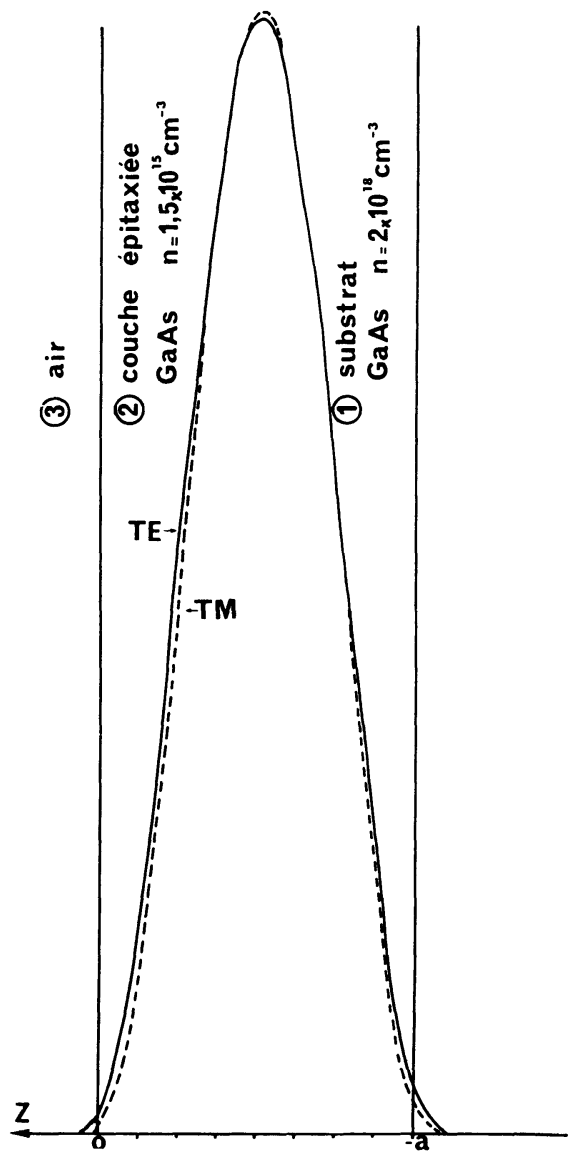

Fig. 4. - Répartition de la densité d'énergie pour les modes $\mathrm{TE}_{0}$ et $\mathrm{TM}_{0}$.

La création de porteurs de densité décroissante de la surface vers l'intérieur suivant une loi en $\exp (+\gamma z)$ modifie les constantes de propagation. Sur la figure 3 apparaitt, en fonction de la densité de porteurs injectés, la variation de la partie imaginaire de la constante de propagation (pertes de propagation). Sans injection, les pertes résiduelles sont dues au substrat. Lorsqu'on injecte des porteurs, ces pertes croissent. Les trois courbes proposées correspondent à trois valeurs de $\gamma$. Les points expérimentaux, obtenus à l'aide du montage décrit ci-dessus, indiquent une profondeur de pénétration des porteurs de l'ordre de 2,5 $\mu \mathrm{m}$. La vitesse de propagation est aussi modifiée (d'une unité de la $3^{\mathrm{e}}$ décimale dans le cas présent).

4. Montage expérimental (Fig. 5). - La photoinjection des porteurs $[2,3,4]$ est réalisée à l'aide d'un 




Fig. 5. - Schéma de principe du montage expérimental. 1) Oscillographe large bande. 2) Laser à rubis déclenché.

3) Atténuateur optique. 4) Cellule photoélectrique. 5) Fente.

6) Guide. 7) Cellule $\mathrm{HgTe}-\mathrm{CdTe}$. 8) Laser $\mathrm{CO}_{2}$ continu. 9) Atténuateur variable.

laser à rubis déclenché fournissant des impulsions de $40 \mathrm{~ns}$, de puissance comprise entre 0 et $10 \mathrm{~kW} / \mathrm{cm}^{+2}$ à l'aide d'un jeu d'atténuateurs. Les porteurs créés (environ $10^{20}$ paires par $\mathrm{cm}^{3}$ ) sur une profondeur de l'ordre de $0,5 \mu \mathrm{m}$ diffusent dans la masse avant d'être recombinés. L'onde de surface à $10,6 \mu \mathrm{m}$ lit leur présence et suit leur répartition. Sur la figure 6 , on peut

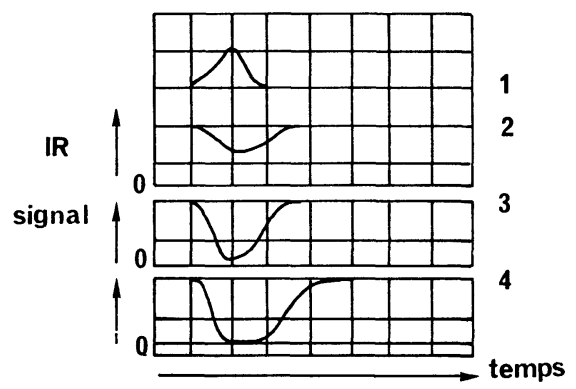

Fig. 6. - Exemple d'oscillogramme : 1) Impulsion excitatrice. Modulation de l'onde guidée : 2) à intensité excitatrice faible, 3) à intensité excitatrice moyenne, 4) à intensité excitatrice forte.

voir (ligne du haut) l'intensité excitatrice à $0,69 \mu \mathrm{m}$ et la transmission à $10,6 \mu \mathrm{m}$ pour trois valeurs de l'intensité excitatrice. L'aspect de l'ensemble de ces courbes s'explique par un temps de vie des porteurs de l'ordre de $25 \times 10^{-8} \mathrm{~s}$. Les études actuelles, reprises avec une excitation plus brève à longueur d'onde variable, constitueront une caractérisation plus complète du semiconducteur (temps de vie en volume, vitesse de recombinaison superficielle, temps de collision, densité de pièges) [5]. Si besoin était, le modèle théorique se prêterait à une stratification plus fine de la structure permettant de faire varier les paramètres électroniques dans l'épaisseur de la couche épitaxiée.

5. Conclusion. - Nous avons montré expérimentalement et théoriquement la possibilité de perturber une onde infrarouge guidée à la surface d'un semi-conducteur grâce à la variation d'indice complexe liée à un gradient de concentration de porteurs libres. Dans l'étape en cours de développement, nous utiliserons cette méthode comme un moyen de caractérisation de semi-conducteurs en couche épitaxiée, caractérisation difficile à obtenir par les méthodes électriques. Nous aurons au passage à notre disposition un moyen électrooptique très puissant de modulation en phase et en amplitude d'une onde infrarouge à l'aide d'une puissance de commande très faible $(\sim 100 \mathrm{~mW})$.

\section{ANNEXE I}

Guide à 3 milieux homogènes. - En mode TE comme en mode TM, l'équation de propagation est de la forme :

$$
\frac{\partial^{2} V}{\partial z^{2}}+\left[\frac{\omega^{2}}{c^{2}} \varepsilon-\beta^{2}\right] V=0
$$

avec :

$$
\begin{aligned}
\omega= & \text { pulsation optique } \\
\varepsilon= & \text { constante diélectrique complexe du milieu dans } \\
& \text { chacun des } 3 \text { milieux considérés, } \\
c= & \text { vitesse de la lumière } \\
\beta= & \text { vecteur de propagation complexe dans la direc- } \\
& \text { tion } x .
\end{aligned}
$$

Nous obtenons ainsi les composantes transversales pour chacun des milieux :

$V_{1 y}=A_{1} \exp \left(j k_{1} z\right) \exp j(\omega t-\beta x)$

$V_{2 y}=\left[A_{2} \exp \left(j k_{2} z\right)+B_{2} \exp -\left(j k_{2} z\right)\right] \exp j(\omega t-\beta x)$ $V_{3 y}=B_{3} \exp -\left(j k_{3} z\right) \exp j(\omega t-\beta x)$.

Les parties imaginaires de $k_{1}$ et $k_{3}$ étant négatives pour que les champs s'annulent à l'infini.

Les constantes $A_{1}, A_{2}, B_{2}$ et $B_{3}$ sont éliminées à partir des conditions de continuité du champ électromagnétique à la surface de séparation de chacun des milieux.

En posant :

$$
K^{2}=\frac{\omega^{2}}{c^{2}} \varepsilon
$$

et

$$
k^{2}=K^{2}-\beta^{2} .
$$

Nous obtenons les équations suivantes :

$\exp 2 j k_{2} a=\frac{\frac{k_{2}}{\varepsilon_{2}}-\frac{k_{1}}{\varepsilon_{1}}}{\frac{k_{2}}{\varepsilon_{2}}+\frac{k_{1}}{\varepsilon_{1}}} \cdot \frac{\frac{k_{2}}{\varepsilon_{2}}-\frac{k_{3}}{\varepsilon_{3}}}{\frac{k_{2}}{\varepsilon_{2}}+\frac{k_{3}}{\varepsilon_{3}}} \quad$ (mode TM)

$\exp 2 j k_{2} a=\frac{k_{2}-k_{1}}{k_{2}+k_{1}} \cdot \frac{k_{2}-k_{3}}{k_{2}+k_{3}} \quad$ (mode TE). 


\section{ANNEXE II}

Evolution des modes d'un guide avec une photoperturbation. - La constante diélectrique d'un milieu dans lequel sont créés des paires électron-trou s'écrit selon la théorie de Drude-Lorentz:

$$
\varepsilon^{\prime}=\varepsilon-\frac{N(z) e^{2}}{m_{\mathrm{e}} \varepsilon_{0} \omega} \cdot \frac{1}{\omega-j \omega_{\mathrm{ce}}}-\frac{N(z) e^{2}}{m_{\mathrm{p}} \varepsilon_{0} \omega} \cdot \frac{1}{\omega-j \omega_{\mathrm{cp}}}
$$

$\varepsilon=$ constante diélectrique complexe avant perturbation,

$N(z)=$ densité des paires créées dans le matériau, $m_{\mathrm{e}}=$ masse efficace des électrons libres,

$m_{\mathrm{p}}=$ masse efficace des trous,

$\omega_{\mathrm{ce}}=$ pulsation de collision des électrons libres, $\omega_{\mathrm{cp}}=$ pulsation de collision des trous.

Si $\omega_{\mathrm{ce}}$ et $\omega_{\mathrm{cp}}$ sont petits devant $\omega$ (c'est le cas pour une onde infrarouge $10,6 \mu \mathrm{m}$ dans l'arséniure de gallium), la constante diélectrique s'écrit :

$$
\varepsilon^{\prime}=\varepsilon-\frac{N(z) e^{2}}{m \varepsilon_{0} \omega} \cdot \frac{1}{\omega-j \omega_{c}}
$$

avec

$$
\frac{1}{m}=\frac{1}{m_{\mathrm{e}}}+\frac{1}{m_{\mathrm{p}}}
$$

et

$$
\omega_{\mathrm{c}}=\frac{m}{m_{\mathrm{e}}} \omega_{\mathrm{ce}}+\frac{m}{m_{\mathrm{p}}} \omega_{\mathrm{cp}} .
$$

Nous envisageons deux types de distribution :

1) Répartition homogène dans une couche d'épaisseur $\delta$ (Fig. $7 a)$; le guide est alors constitué de 4 milieux
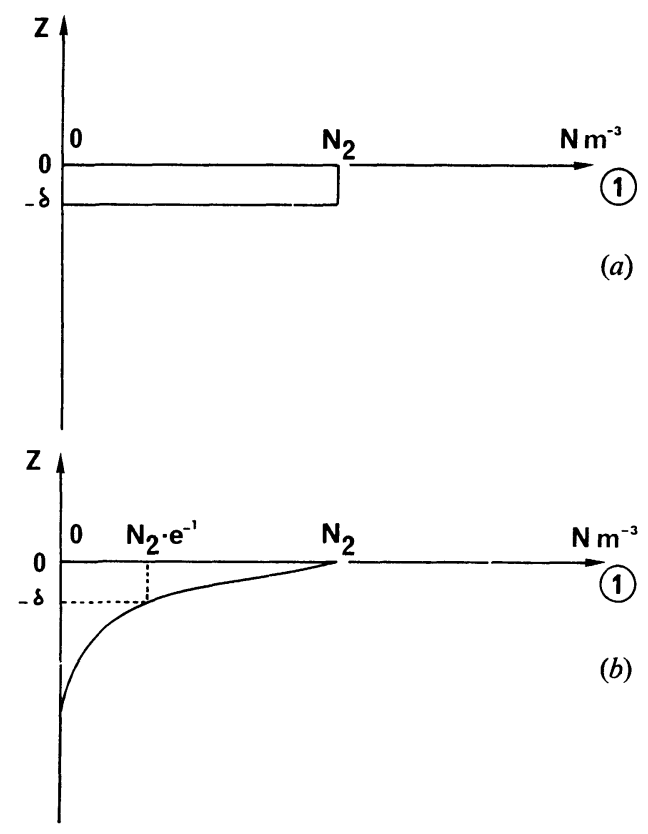

FIG. 7. - Répartition de la concentration volumique des photoporteurs dans le guide perturbé. a) Répartition homogène ; b) Répartition exponentielle. homogènes. Un calcul analogue à celui de l'annexe I conduit à l'expression :

$$
\begin{array}{r}
\frac{k_{2}}{\varepsilon_{2}} \cdot \frac{\left(\frac{k_{2}}{\varepsilon_{2}}+\frac{k_{1}}{\varepsilon_{1}}\right)-\left(\frac{k_{2}}{\varepsilon_{2}}-\frac{k_{1}}{\varepsilon_{1}}\right) \exp -\left[2 j k_{2}(a-\delta)\right]}{\left(\frac{k_{2}}{\varepsilon_{2}}+\frac{k_{1}}{\varepsilon_{1}}\right)+\left(\frac{k_{2}}{\varepsilon_{2}}-\frac{k_{1}}{\varepsilon_{1}}\right) \exp -\left[2 j k_{2}(a-\delta)\right]}= \\
=\frac{k_{2}^{\prime}}{\varepsilon_{2}^{\prime}} \frac{\left(\frac{k_{2}^{\prime}}{\varepsilon_{2}^{\prime}}-k_{3}\right)-\left(\frac{k_{2}^{\prime}}{\varepsilon_{2}^{\prime}}+k_{3}\right) \exp \left(2 j k_{2}^{\prime} \delta\right)}{\left(\frac{k_{2}^{\prime}}{\varepsilon_{2}^{\prime}}-k_{3}\right)+\left(\frac{k_{2}^{\prime}}{\varepsilon_{2}^{\prime}}+k_{3}\right) \exp \left(2 j k_{2}^{\prime} \delta\right)}
\end{array}
$$

avec

$$
k_{2}^{\prime 2}=K_{2}^{\prime 2}-K_{2}^{2}+k_{2}^{2}
$$

pour le mode TM.

En considérant des milieux de même perméabilité magnétique, le résultat pour le mode TE s'obtient en remplaçant $\varepsilon_{1}, \varepsilon_{2}, \varepsilon_{2}^{\prime}$ par 1 dans l'expression ci-dessus.

2) Distribution exponentielle :

$$
N(z)=N_{2} \mathrm{e}^{y z} \quad \text { (Fig. 7b). }
$$

Dans les milieux 1 et 3 les équations sont du type défini dans l'annexe I, leurs solutions restent donc des ondes planes.

Dans le milieu 2 l'équation à résoudre s'écrit :

$$
\frac{\partial^{2} V_{2}}{\partial z^{2}}+\frac{\omega^{2}}{c^{2}}\left[\varepsilon_{2}-\rho_{2} \mathrm{e}^{\gamma z}-b\right] V_{2}=0
$$

avec :

$$
\rho_{2}=\frac{N_{2} e^{2}}{m \varepsilon_{0} \omega} \times \frac{1}{\omega-j \omega_{c}} \quad b=\frac{c^{2} \beta^{2}}{\omega^{2}} .
$$

En posant :

$$
\begin{aligned}
& Z=\frac{2 j \omega \sqrt{\rho_{2}}}{\gamma c} \mathrm{e}^{\gamma z / 2} \\
& \nu=\frac{2 j \omega}{\gamma c} \sqrt{\varepsilon_{2}-b}
\end{aligned}
$$

l'équation prend la forme :

$$
Z^{2} V_{Z}^{\prime \prime}+Z V_{Z}^{\prime}+\left(Z^{2}-v^{2}\right) V=0
$$

soit

$$
V_{2 y}=A J_{v}(Z)+B J_{-v}(Z)
$$

$J_{v}$ et $J_{-v}$ étant des fonctions de Bessel de $1^{\text {re }}$ espèce à variable et indice complexes.

Les conditions de continuités conduisent à une équation où $b$ est l'inconnue. 
Pour le mode TM :

$\frac{\left(\varepsilon_{20} \sqrt{b-1}+\sqrt{b-\varepsilon_{2}}\right) J_{v}\left(Z_{0}\right)-j \sqrt{\rho_{2}} J_{v+1}\left(Z_{a}\right)}{\left(\varepsilon_{2 a} \sqrt{b-\varepsilon_{1}}-\varepsilon_{10} \sqrt{b-\varepsilon_{2}}\right) J_{v}\left(Z_{a}\right)+j \varepsilon_{10} \sqrt{\rho_{2}} \mathrm{e}^{-\gamma a / 2} J_{v+1}\left(Z_{a}\right)}=$

$$
=\frac{\left(\varepsilon_{20} \sqrt{b-1}+\sqrt{b-\varepsilon_{2}}\right) J_{-v}\left(Z_{0}\right)+j \sqrt{\rho_{2}} J_{-(v+1)}\left(Z_{0}\right)}{\left(\varepsilon_{2 a} \sqrt{b-\varepsilon_{1}}-\varepsilon_{10} \sqrt{b-\varepsilon_{2}}\right) J_{-v}\left(Z_{a}\right)-j \varepsilon_{10} \sqrt{\rho_{2}} \mathrm{e}^{-\gamma a / 2} J_{-(v+1)}\left(Z_{a}\right)}
$$

avec

$$
\begin{array}{ll}
\varepsilon_{20}=\varepsilon_{2}-\rho_{2} ; & \varepsilon_{2 a}=\varepsilon_{2}-\rho_{2} \mathrm{e}^{-\gamma a} \\
Z_{0}=\frac{2 j \omega \sqrt{\rho_{2}}}{\gamma c} ; & Z_{a}=Z_{0} \mathrm{e}^{-\gamma a / 2} .
\end{array}
$$

Pour le mode TE il suffit de remplacer $\varepsilon_{20}, \varepsilon_{2 a}, \varepsilon_{10}$ par 1 dans l'équation précédente.

\section{Bibliographie}

[1] Sitzer, W. G. and FAN, H. Y., Phys. Rev. 106 (1957) 882.

[2] Blinov, L. M., Vavilov, V. S. et Galkin, G. N., Fiz. Tekh. Poluprovodn 1 (1967) 1351.

[3] Mc Fee, J. H., Nahory, R. E., Pollack, M. A. and LOGAN, R. A., $A P L 23$ (1973) 571

[4] Botineau, J., Gires, F. et Vanneste, C., C. R. Hebd. Séan. Acad. Sci. 278 (1973) 171.

[5] Azema, A., Botineau, J., Gires, F., Saissy, A. et Vanneste, C., Appl. Phys. 9 (1976) 47. 